\section{BRICNET, UMA REDE BRASILEIRA COLABORATIVA PARA A REALIZAÇÃO DE ESTUDOS MULTICÊNTRI- COS EM MEDICINA INTENSIVA.}

\author{
BRICNET, A COLLABORATIVE BRAZILIAN NETWORK \\ TO CONDUCT AND TO PROMOTE MULTICENTER \\ STUDIES IN INTENSIVE CARE.
}

Senhor Editor,

É com grande satisfação que apresentamos a Brazilian Research in Intensive Care Network - BRICNet, uma rede brasileira, independente e colaborativa para a realização de estudos multicêntricos na área de Medicina Intensiva.

Os centros brasileiros têm contribuído significativamente para a realização de estudos multicêntricos internacionais e a participação do Brasil em estudos recentes é exemplo do nosso potencial para a realização de estudos multicêntricos próprios de qualidade.

O nome BRICNet teve como inspiração a sigla atribuída aos países considerados como economias promissoras (BRIC, de Brasil, Rússia, Índia e China), em que a expectativa é de que se tornem potências econômicas mundiais em um futuro muito próximo. De modo análogo, vemos o Brasil como uma potência emergente na produção de conhecimento científico, em particular, na área de Medicina intensiva e o momento atual é especialmente propício para a criação e consolidação de uma rede colaborativa. Neste contexto, tomamos a iniciativa de criar a BRICNet, que esperamos possa servir para chancelar, conduzir e apoiar a realização destes estudos em cooperação. Redes como a BRICNet foram fundamentais para o fortalecimento das relações entre as instituições, para a melhoria da qualidade dos estudos e para o incremento da produção científica na área da Medicina Intensiva em países como o Canadá, Austrália, França e Inglaterra.

O comitê coordenador da rede é constituído de pesquisadores renomados na área de Medicina Intensiva vinculados a instituições de ensino, pesquisa e assistência de diversas regiões do nosso país sob a chancela do Instituto Nacional de Câncer, um órgão do Ministério da Saúde. A rede dispõe do endereço eletrônico - www.bricnet.org - onde as informações pertinentes podem ser obtidas - e está cadastrada no Diretório Grupos de Pesquisa do CNPq.

A BRICNet tem como missões promover e chancelar a realização de estudos clínicos multicêntricos em pacientes criticamente enfermos que reflitam a realidade do Brasil, e fortalecer as relações entre as instituições e centros participantes. A rede pretende atuar de forma ética, transparente e democrática, promovendo estudos próprios, em princípio dois a cada ano, e também chancelando estudos multicêntricos na área de Terapia Intensiva que tenham abrangência nacional, e relevância científica e para a melhoria dos cuidados dos pacientes criticamente enfermos.

O primeiro estudo, intitulado "Estudo multicêntrico sobre a avaliação do prognóstico de pacientes com câncer, internados em unidades de terapia intensiva" já está em curso e conta com a participação de 45 centros de 17 estados das cinco regiões geográficas brasileiras e a estimativa é que 1500 a 1800 pacientes sejam incluídos.

Esperamos que uma rede independente de cooperação desse porte propicie condições mais apropriadas para a condução e chancela de estudos multicêntricos com pacientes criticamente enfermos dentro da realidade do Brasil que sejam capazes de fornecer informações que possam ser úteis para a orientação das políticas de saúde pública, para a melhoria dos cuidados dos pacientes e para o incremento da produção científica do Brasil na área da Medicina intensiva.

Atenciosamente,

Comitê Coordenador

BRICNet - Brazilian Research in Intensive Care $\mathrm{Ne}$ twork

Instituto Nacional de Câncer - INCA

Centro de Tratamento Intensivo

Núcleo de Pesquisa Clínica em Medicina Intensiva

Praça Cruz Vermelha, 23 - $10^{\circ}$ Andar

20230-130 Rio de Janeiro, RJ

Tel: +55212506 6120

Fax: +55 2122948620

E-mail: bricnet@yahoo.com.br

Website: www.bricnet.org 